PROCEEDINGS OF THE AMERICAN MATHEMATICAL SOCIETY

Volume 124, Number 10, October 1996

\title{
A QUICK PROOF OF THE CLASSIFICATION OF SIMPLE REAL LIE ALGEBRAS
}

\author{
A. W. KNAPP \\ (Communicated by Roe W. Goodman)
}

\begin{abstract}
Cartan's classification of the simple Lie algebras over $\mathbb{R}$ is derived quickly from some structure theory over $\mathbb{R}$ and the classification over $\mathbb{C}$.
\end{abstract}

Élie Cartan classified the simple Lie algebras over $\mathbb{R}$ for the first time in 1914 . There have been a number of simplifications in the proof since then, and these are described in [3, p. 537]. All proofs assume the classification over $\mathbb{C}$ and a certain amount of structure theory over $\mathbb{R}$. Recent proofs tend to run to 25 pages. Here is a shorter argument.

Theorem. Up to isomorphism, the only simple Lie algebras over $\mathbb{R}$ that are neither complex nor compact are those in Cartan's list as organized in [3, p. 518].

We use terminology as in [3]. Let $\mathfrak{g}_{0}=\mathfrak{k}_{0} \oplus \mathfrak{p}_{0}$ be a Cartan decomposition of a noncomplex simple Lie algebra over $\mathbb{R}$, and let $\theta$ be the Cartan involution. Choose a maximal abelian subspace $\mathfrak{t}_{0}$ of $\mathfrak{k}_{0}$ and extend to a maximally compact Cartan subalgebra $\mathfrak{h}_{0}=\mathfrak{t}_{0} \oplus \mathfrak{a}_{0}$ of $\mathfrak{g}_{0}$. Removal of subscripts 0 will indicate complexifications. Let $\Delta=\Delta(\mathfrak{g}, \mathfrak{h})$ be the root system. Roots are imaginary on $\mathfrak{t}_{0}$ and real on $\mathfrak{a}_{0}$. All roots are imaginary-valued or complex on $\mathfrak{h}_{0}$; there are no real-valued roots. Introduce a positive system $\Delta^{+}$that takes $i \mathfrak{t}_{0}$ before $\mathfrak{a}_{0}$. The map $\theta$ carries roots to roots and permutes the simple roots. The complex simple roots move in two-element orbits, while the imaginary simple roots are fixed. By the Diagram of $\left(\mathfrak{g}_{0}, \mathfrak{h}_{0}, \Delta^{+}\right)$, we mean the Dynkin diagram of $\Delta$ with the two-element orbits under $\theta$ so labeled and with the imaginary roots shaded or not, according as the simple root is noncompact (root vector in $\mathfrak{p}$ ) or compact (root vector in $\mathfrak{k}$ ).

Lemma 1. If $\left(\mathfrak{g}_{0}, \mathfrak{h}_{0}, \Delta^{+}\right)$and $\left(\mathfrak{g}_{0}^{\prime}, \mathfrak{h}_{0}^{\prime},\left(\Delta^{\prime}\right)^{+}\right)$have the same Diagram, then $\mathfrak{g}_{0}$ and $\mathfrak{g}_{0}^{\prime}$ are isomorphic.

Proof. We may assume that the complexifications $\left(\mathfrak{g}, \mathfrak{h}, \Delta^{+}\right)$are the same and that the associated compact forms are the same: $\mathfrak{u}_{0}=\mathfrak{k}_{0} \oplus i \mathfrak{p}_{0}=\mathfrak{k}_{0}^{\prime} \oplus i \mathfrak{p}_{0}^{\prime}$. Using the conjugacy of compact forms, the conjugacy of maximal abelian subspaces within them, and the standard construction of a compact form from $\mathfrak{h}$, we see that we can normalize root vectors $X_{\alpha}, \alpha \in \Delta$, as in Theorem 5.5 of [3, p. 176] and obtain $\mathfrak{u}_{0}$ from $\left\{X_{\alpha}\right\}$ as in Theorem 6.3 of [3, p. 181].

Received by the editors April 12, 1995.

1991 Mathematics Subject Classification. Primary 17B20, 22 E15.

(C)1996 American Mathematical Society 
First suppose $\mathfrak{a}_{0}=0$, so that all roots are imaginary. For $\alpha$ simple we have $\theta X_{\alpha}= \pm X_{\alpha}$, the sign being + if $\alpha$ is compact and - is $\alpha$ is noncompact. The same formula holds for $\theta^{\prime}$. Since $\mathfrak{h}$ and the $X_{\alpha}$ 's for $\alpha$ simple generate $\mathfrak{g}$, it follows that $\theta=\theta^{\prime}$, hence that $\mathfrak{k}=\mathfrak{k}^{\prime}$ and $\mathfrak{p}=\mathfrak{p}^{\prime}$. Then $\mathfrak{g}_{0}=\mathfrak{g}_{0}^{\prime}$ is recovered as $\left(\mathfrak{u}_{0} \cap \mathfrak{k}\right) \oplus i\left(\mathfrak{u}_{0} \cap \mathfrak{p}\right)$.

If $\mathfrak{a}_{0} \neq 0$, we may not have $\theta=\theta^{\prime}$. For $\alpha \in \Delta$, write $\theta X_{\alpha}=a_{\alpha} X_{\theta \alpha}$. Then $a_{\alpha} a_{-\alpha}=1$ and $a_{\alpha} a_{\theta \alpha}=1$. Since $\theta$ maps $\mathfrak{u}_{0} \cap \operatorname{span}\left\{X_{\alpha}, X_{-\alpha}\right\}$ to $\mathfrak{u}_{0} \cap$ $\operatorname{span}\left\{X_{\theta \alpha}, X_{-\theta \alpha}\right\}$, we see that $\bar{a}_{\alpha}=a_{-\alpha}$. Therefore $\left|a_{\alpha}\right|=1$. For each pair of complex simple roots $\alpha$ and $\theta \alpha$, choose square roots $a_{\alpha}^{1 / 2}$ and $a_{\theta \alpha}^{1 / 2}$ whose product is 1. Similarly write $\theta^{\prime} X_{\alpha}=b_{\alpha} X_{\theta \alpha}$ with $\left|b_{\alpha}\right|=1$, and define $b_{\alpha}^{1 / 2}$ and $b_{\theta \alpha}^{1 / 2}$ for $\alpha$ and $\theta \alpha$ simple. Define $H$ and $H^{\prime}$ in $\mathfrak{h} \cap \mathfrak{u}_{0}$ by the conditions that $\alpha(H)=\alpha\left(H^{\prime}\right)=$ 0 for $\alpha$ simple imaginary and that $\exp \left(\frac{1}{2} \alpha(H)\right)=a_{\alpha}^{1 / 2}$, $\exp \left(\frac{1}{2} \theta \alpha(H)\right)=a_{\theta \alpha}^{1 / 2}$, $\exp \left(\frac{1}{2} \alpha\left(H^{\prime}\right)\right)=b_{\alpha}^{1 / 2}$, and $\exp \left(\frac{1}{2} \theta \alpha\left(H^{\prime}\right)\right)=b_{\theta \alpha}^{1 / 2}$ if $\alpha$ and $\theta \alpha$ are complex simple. A little computation shows that $\theta^{\prime} \circ \operatorname{Ad}\left(\exp \frac{1}{2}\left(H-H^{\prime}\right)\right)=\operatorname{Ad}\left(\exp \frac{1}{2}\left(H-H^{\prime}\right)\right) \circ \theta$, from which it follows that $\mathfrak{k}^{\prime}=\operatorname{Ad}\left(\exp \frac{1}{2}\left(H-H^{\prime}\right)\right) \mathfrak{k}, \mathfrak{p}^{\prime}=\operatorname{Ad}\left(\exp \frac{1}{2}\left(H-H^{\prime}\right)\right) \mathfrak{p}$, and $\mathfrak{g}_{0}^{\prime}=\operatorname{Ad}\left(\exp \frac{1}{2}\left(H-H^{\prime}\right)\right) \mathfrak{g}_{0}$.

The next step is to identify some pairs of distinct Diagrams that correspond merely to changes of $\Delta^{+}$. The argument is inspired by [2]. First let us assume that $\mathfrak{a}_{0}=0$, i.e., that the automorphism of $\Delta$ given by $\theta$ is the identity. Let $\Lambda$ be the subset of $i \mathrm{t}_{0}$ where all roots take integer values and where all noncompact roots take odd-integer values. If $\left\{\omega_{j}\right\}$ is the basis dual to the simple roots, then the sum of those $\omega_{j}$ corresponding to the noncompact simple roots is a member of $\Lambda$. The set $\Lambda$ is discrete, and we let $H_{0}$ be a member of $\Lambda$ as close to 0 as possible.

Lemma 2. If $\left(\Delta^{+}\right)^{\prime}$ is a positive system that makes $H_{0}$ dominant, then there is at most one noncompact simple root, say $\alpha_{i}$. If the basis dual to the simple roots of $\left(\Delta^{+}\right)^{\prime}$ is $\left\{\omega_{j}\right\}$, then there cannot exist $i^{\prime}$ such that $\left\langle\omega_{i}-\omega_{i^{\prime}}, \omega_{i^{\prime}}\right\rangle>0$.

Proof. Since $H_{0}$ is in $\Lambda$ and is dominant, $H_{0}=\sum n_{j} \omega_{j}$ with all $n_{j}$ integers $\geq 0$. If $n_{i}>0$, then $H_{0}-\omega_{i}$ is dominant and thus has $\left\langle H_{0}-\omega_{i}, \omega_{i}\right\rangle \geq 0$ with equality if and only if $H_{0}=\omega_{i}$. Then $\left|H_{0}-2 \omega_{i}\right|^{2} \leq\left|H_{0}\right|^{2}$ with equality only if $H_{0}=\omega_{i}$, and minimality forces $H_{0}=\omega_{i}$. Now let $H_{0}=\omega_{i}$. If $\left\langle\omega_{i}-\omega_{i^{\prime}}, \omega_{i^{\prime}}\right\rangle>0$, then $\left|H_{0}-2 \omega_{i^{\prime}}\right|^{2}<\left|H_{0}\right|^{2}$, in contradiction to minimality.

When $\mathfrak{a}_{0} \neq 0$, Lemma 2 is to be applied to the part of $i \mathfrak{t}_{0}$ corresponding to the span of the imaginary simple roots. The result is that we can associate to any $\mathfrak{g}_{0}$ at least one Diagram in which at most one imaginary root is shaded.

Now we can read off the possibilities. First suppose that the automorphism of $\Delta$ is the identity. If all roots are unshaded, then $\mathfrak{g}_{0}$ is the compact form. Otherwise exactly one simple root is shaded. For the classical Dynkin diagrams, let the double line or triple point be at the right end, and let the $i^{\text {th }}$ root be shaded. In $A_{n}$, we are led to $\mathfrak{s u}(i, n+1-i)$. In $B_{n}$, we are led to $\mathfrak{s o}(2 i, 2 n+1-2 i)$. In $C_{n}$, we are led to $\mathfrak{s p}(i, n-i)$ if $i<n$ and to $\mathfrak{s p}(n, \mathbb{R})$ if $i=n$. In $D_{n}$, we are led to $\mathfrak{s o}(2 i, 2 n-2 i)$ if $i \leq n-2$ and to $\mathfrak{s o}^{*}(2 n)$ otherwise.

For the exceptional Dynkin diagrams, a little checking that compares the second conclusion of Lemma 2 with the fundamental weights (see [1, pp. 260-275]) shows that $\alpha_{i}$ in Lemma 2 has to be a node (endpoint vertex) of the Dynkin diagram. Moreover, in $G_{2}, \alpha_{i}$ has to be the long simple root, while in $E_{8}$, it cannot be the node on the short branch. In $E_{6}$ two nodes are equivalent by outer automorphism. Thus we obtain at most three Lie algebras for $E_{7}$; at most two for $E_{6}, E_{8}, F_{4}$; and 
at most one for $G_{2}$. These are E II, E III for $E_{6}, \mathrm{E} \mathrm{V}, \mathrm{E}$ VI, E VII for $E_{7}$; E VIII, E IX for $E_{8}$; F I, F II for $F_{4}$; and $G$ for $G_{2}$.

When the automorphism of $\Delta$ is not the identity, the Dynkin diagram is $A_{n}, D_{n}$, or $E_{6}$. For $A_{n}$, there is no imaginary simple root if $n$ is even, and there is one if $n$ is odd. For $n$ even we are led to $\mathfrak{s l}(n+1, \mathbb{R})$, while for $n$ odd we are led to $\mathfrak{s l}(n+1, \mathbb{R})$ if the root is shaded and to $\mathfrak{s u}^{*}(n+1)$ if the root is unshaded. For $D_{n}$, the first $n-2$ simple roots are imaginary. If all are unshaded, we are led to $\mathfrak{s o}(1,2 n-1)$. If the $i^{\text {th }}$ simple root is shaded, $i \leq n-2$, we are led to $\mathfrak{s o}(2 i+1,2 n-2 i-1)$. For $E_{6}$, the triple point and the node on the short branch are imaginary. If neither is shaded, we are led to E IV, while if either one is shaded, we are led to E I.

Note added in proof. David Vogan has pointed out that any Dynkin diagram marked with an involution and having a subset of its one-element orbits shaded is a Diagram for some $\mathfrak{g}_{0}$. The proof is in the spirit of Lemma 1. Existence of the exceptional simple real Lie algebras follows.

\section{REFERENCES}

1. N. Bourbaki, Groupes et Algèbres de Lie, Chapitres 4, 5, et 6, Hermann, Paris, 1968. MR 39:1590

2. A. Borel and J. De Siebenthal, Les sous-groupes fermés de rang maximum des groupes de Lie clos, Comment. Math. Helvetici 23 (1949), 200-221. MR 11:326d

3. S. Helgason, Differential Geometry, Lie Groups, and Symmetric Spaces, Academic Press, New York, 1978. MR 80k:53081

Department of Mathematics, State University of New York, Stony Brook, New York 11794

E-mail address: aknapp@ccmail.sunysb.edu 